\title{
Review of: "Structural characteristics and proximity comparison of China's urban innovation cooperation network"
}

Xinyuan Liang

Potential competing interests: The author(s) declared that no potential competing interests exist.

Exploring the evolution characteristics of regional urban innovation cooperation networks can help to clarify urban innovation development. After reading the full text, it can be seen that the article has applied a large number of research methods to analyze the characteristics of China's urban innovation cooperation network. Overall, the logic of the article is clear, and the figures are beautifully made. However, there is still much room for improvement before publication. In particular, the expression of scientific questions is not clear enough. For example, why do we need to study China's urban innovation network, and what guiding significance does the study have. Neither in the literature review part nor the analysis of the results, the article did not directly answer. Therefore, the author must clearly state the value of the research so that readers can understand the concept and the practical significance of the research. Detailed opinions:

There should be no words with regional attributes, e.g., new normal, in ABSTRACT. So what does this word mean?

There is too much content in INTRODUCTION, and its logical expression should be reorganized. The first paragraph is not suitable as an opening paragraph. Suggestions can be carried out in the order of comprehensive research background, international research hotspots, the necessity of regional research, literature review, research goals and ideas. In addition, the introduction contains many theoretical introductions (such as the fifth paragraph), which can be further summarized as a separate chapter combined with the research ideas of this article.

"self-contained centrality" needs further explanation. Compared with data application, it may be a direct manifestation of the innovation of this article.

In INTRODUCTION, the innovative value positioning of the article is not clear. In my opinion, the application of data and methods cannot be regarded as an innovation point. The most likely innovation in this article lies in theory and analytical thinking. It is recommended to supplement the theoretical framework and analysis chapters, discuss "self-contained centrality", and propose hypotheses about "innovation networks evolution".

For materials and methods, is it feasible to use only patent data to represent urban innovation? The author needs to add an explanation in the acticle.

As a technical route, the expression in Figure 2 is somewhat simple. 
In RESULT analysis, Figure 6 is a long-term series data. Why is there only one picture? All data calculations in the administrative unit should be explained.

The methodology description should be placed in Materials and Methods, such as part of the text description of P12 and P13.

CONCLUSION is verbose and can be reduced appropriately to express the core point of view. Some content looks more like analysis than the conclusion.

DISCUSSION should focus on the uncertainty analysis and applicability of the research method. Besides, it can be compared with other related studies to illustrate this article's practical and theoretical research value. 